\title{
On a theorem of Avez
}

\author{
Murray Elder and Cameron Rogers \\ Communicated by George Willis
}

\begin{abstract}
For each symmetric, aperiodic probability measure $\mu$ on a finitely generated group $G$, we define a subset $A_{\mu}$ consisting of group elements $g$ for which the limit of the ratio $\mu^{* n}(g) / \mu^{* n}(e)$ tends to 1 . We prove that $A_{\mu}$ is a subgroup, is amenable, contains every finite normal subgroup, and $G=A_{\mu}$ if and only if $G$ is amenable. For non-amenable groups we show that $A_{\mu}$ is not always a normal subgroup and can depend on the measure. We formulate some conjectures relating $A_{\mu}$ to the amenable radical.
\end{abstract}

\section{Introduction}

Let $\mu$ be a symmetric, aperiodic probability measure $\mu$ on a finitely generated group $G$ whose support generates $G$. Let $e$ denote the identity element of $G$, and let $\mu^{* n}$ denote the $n$-fold convolution of the measure, so that $\mu^{* n}(g)$ is the probability that an $n$-step random walk induced by $\mu$ starting at $e$ ends at $g$. Avez [2] showed that when $G$ is amenable,

$$
\lim _{n \rightarrow \infty} \frac{\mu^{* n}(g)}{\mu^{* n}(e)}=1 \quad \text { for all } g \in G .
$$

In this paper, we extend Avez' result in the following way: For an arbitrary finitely generated group $G$, we consider the set, which we call $A_{\mu}$, of all $g \in G$ for which the limit of the ratio $\mu^{* n}(g) / \mu^{* n}(e)$ tends to 1 . Avez' result says that if $G$ is amenable, then $A_{\mu}=G$. We prove that when $G$ is non-amenable, $A_{\mu}$ is a proper, amenable subgroup. Moreover, $A_{\mu}$ contains every finite normal subgroup, so contains the elliptic radical (the largest normal, locally finite subgroup of $G$ ), and so is non-trivial in many cases. We compute $A_{\mu}$ for some examples and show that, in general, it is not a normal subgroup and may depend on the measure. We close by formulating some conjectures relating $A_{\mu}$ to the amenable radical.

This work is part of PhD work of the second author [13]; more details and applications can be found therein. Other relevant work that motivates the present paper includes $[3,7-10,12,14]$.

Research supported by Australian Research Council grant FT110100178. 


\section{Preliminaries}

In this article, $\mathbb{Z}_{+}$denotes the positive integers. Recall that a probability measure $\mu$ on a group $G$ is symmetric if $\mu(x)=\mu\left(x^{-1}\right)$ for all $x \in G$. The support of $\mu$ is the set $\{x \in G \mid \mu(x)>0\}$, which we denote by $\operatorname{supp}(\mu)$. The convolution $\mu * \tau$ of two measures $\mu, \tau$ on a discrete group is

$$
\mu * \tau(y)=\sum_{x \in G} \mu(x) \tau\left(x^{-1} y\right) .
$$

The distribution of a $n$-step random walk induced by $\mu$ is the $n$-fold convolution power of $\mu$, which we denote by $\mu^{* n}$. The period of a measure $\mu$ is

$$
\operatorname{gcd}\left\{n \in \mathbb{Z}_{+} \mid \mu^{* n}(e)>0\right\} .
$$

The measure $\mu$ is said to be aperiodic if it has period 1 . Note that for a symmetric measure, the period can only take the values 1 or 2 .

A function $\zeta: G \rightarrow \mathbb{R}$ on a finitely generated group $G$ is an $\ell^{2}$-function, or $\zeta \in \ell^{2}(G)$, if $\sum_{g \in G}|\zeta(g)|^{2}$ is finite. The corresponding inner product is

$$
\langle\zeta, \iota\rangle_{2}=\sum_{g \in G} \zeta(g) \iota(g),
$$

and the norm is $\|\zeta\|_{2}=\sqrt{\langle\zeta, \zeta\rangle}$, as usual. The action of the group $G$ on $\ell^{2}(G)$ defined by $g \cdot \zeta(x)=\zeta\left(g^{-1} x\right)$ for all $x \in G$ is called the left regular representation of the group.

Observe that

$$
\begin{aligned}
\mu^{* 2 n}(g) & =\sum_{x \in G} \mu^{* n}(x) \mu^{* n}\left(x^{-1} g\right) \\
& =\sum_{x \in G} \mu^{* n}(x) \mu^{* n}\left(g^{-1} x\right) \\
& =\sum_{x \in G} \mu^{* n}(x)\left(g \cdot \mu^{* n}(x)\right) \\
& =\left\langle\mu^{* n}, g \cdot \mu^{* n}\right\rangle,
\end{aligned}
$$

and so $\mu^{* 2 n}(e)=\left\langle\mu^{* n}, \mu^{* n}\right\rangle=\left\|\mu^{* n}\right\|_{2}^{2}$.

The notion of amenability has many characterizations. Here we use the following:

Theorem 2.1 ([5,11]). $G$ is amenable if and only if there is a sequence $f_{n}$ of probability measures on $G$ such that $\left\|g \cdot f_{n}-f_{n}\right\|_{2} \rightarrow 0$ for every $g \in G$. 


\section{Defining $A_{\mu}$}

Definition 3.1. Let $G$ be a finitely generated group, and let $\mu$ be a symmetric, aperiodic probability measure on $G$ whose support generates $G$. We define

$$
A_{G, \mu}=\left\{\begin{array}{l|l}
g \in G & \lim _{n \rightarrow \infty} \frac{\mu^{* n}(g)}{\mu^{* n}(e)}=1
\end{array}\right\} .
$$

When it is understood which group is being used, the set will be referred to as $A_{\mu}$.

The definition is clearly motivated by Avez' result: when $G$ is amenable, we have $A_{\mu}=G$. A similar construction based on Theorem 2.1 would be the set of all $g \in G$ for which $\left\|g \cdot f_{n}-f_{n}\right\|_{2}$ tends to 0 with respect to some fixed sequence $f_{n}$ of probability measures on $G$. An obvious choice for such a sequence would be $\xi_{n}=\frac{\mu^{* n}}{\left\|\mu^{* n}\right\|_{2}}$. It turns out that this construction coincides with $A_{\mu}$.

Proposition 3.2. Let $G$ be a finitely generated group and $\mu$ a symmetric, aperiodic probability measure on $G$ whose support generates $G$. Then

$$
A_{\mu}=\left\{g \in G \mid\left\|g \cdot \xi_{n}-\xi_{n}\right\|_{2} \rightarrow 0\right\} .
$$

Proof. By equation (2.1), we have

$$
\frac{\mu^{* 2 n}(g)}{\mu^{* 2 n}(e)}=\frac{\left\langle\mu^{* n}, g \cdot \mu^{* n}\right\rangle}{\left\|\mu^{* n}\right\|_{2}^{2}}=\left\langle\xi_{n}, g \cdot \xi_{n}\right\rangle .
$$

Observe that

$$
\begin{aligned}
\left\|g \cdot \xi_{n}-\xi_{n}\right\|_{2}^{2} & =\sum_{x \in G}\left(g \cdot \xi_{n}-\xi_{n}\right)^{2}(x) \\
& =\sum_{x \in G}\left(g \cdot \xi_{n}\right)^{2}(x)-2 \sum_{x \in G}\left(g \cdot \xi_{n}\right)(x) \xi_{n}(x)+\sum_{x \in G}\left(\xi_{n}\right)^{2}(x) \\
& =\left\|g \cdot \xi_{n}\right\|_{2}^{2}-2\left\langle g \cdot \xi_{n}, \xi_{n}\right\rangle+\left\|\xi_{n}\right\|_{2}^{2} \\
& =2-2\left\langle g \cdot \xi_{n}, \xi_{n}\right\rangle
\end{aligned}
$$

since $\xi_{n}, g \cdot \xi_{n}$ are unit vectors. Thus $\left\|g \cdot \xi_{n}-\xi_{n}\right\|_{2}$ approaches 0 if and only if $\left\langle g \cdot \xi_{n}, \xi_{n}\right\rangle=\frac{\mu^{* 2 n}(g)}{\mu^{* 2 n}(e)}$ approaches 1 .

Corollary 3.3. $G$ is amenable if and only if $G=A_{\mu}$.

Proof. This follows immediately from Theorem 2.1 and Proposition 3.2.

The following observation will be useful.

Lemma 3.4. Let $\mu$ be a symmetric, aperiodic probability measure on $G$ whose support generates $G$. For any fixed $k \in \mathbb{Z}_{+}$, we have $A_{\mu}=A_{\mu * k}$. 


\section{Algebraic properties of $\boldsymbol{A}_{\mu}$}

We now show that more than being some peculiar collection of elements, the sets $A_{\mu}$ have algebraic structure. Throughout this section, we consider $G$ a finitely generated group and $\mu$ a symmetric, aperiodic probability measure on $G$ whose support generates $G$, and $\xi_{n}=\frac{\mu^{* n}}{\left\|\mu^{* n}\right\|_{2}}$.

Theorem 4.1. $A_{\mu}$ is a subgroup.

Proof. Let $g, h \in G$. We have

$$
\begin{aligned}
\left\|g h \cdot \xi_{n}-\xi_{n}\right\|_{2} & =\left\|g \cdot\left(h \cdot \xi_{n}-\xi_{n}\right)+g \cdot \xi_{n}-\xi_{n}\right\|_{2} \\
& \leq\left\|g \cdot\left(h \cdot \xi_{n}-\xi_{n}\right)\right\|_{2}+\left\|g \cdot \xi_{n}-\xi_{n}\right\|_{2} \\
& =\left\|h \cdot \xi_{n}-\xi_{n}\right\|_{2}+\left\|g \cdot \xi_{n}-\xi_{n}\right\|_{2}
\end{aligned}
$$

since the $\ell^{2}$-norm is invariant under translation. Since $g, h \in A_{\mu}$ the right-hand side limits to 0 , so $g h \in A_{\mu}$. Clearly, $e \in A_{\mu}$ and $A_{\mu}$ is closed under inverses since $\mu$ is symmetric.

In [13], a slightly stronger statement is given, which gives some structural information about the cosets of $A_{\mu}$.

Theorem 4.2. $A_{\mu}$ is amenable.

The idea of our proof is to give a sequence of probability measures on $A_{\mu}$ that are almost invariant under the action of $A_{\mu}$. Proposition 3.2 says that we have such a sequence in $\ell^{2}(G)$, which we modify to obtain a sequence in $\ell^{2}\left(A_{\mu}\right)$.

Proof. Choose a set $I=\left\{s_{1}, s_{2}, \ldots\right\}$ of right coset representatives for $A_{\mu}$, which is countable since $G$ is finitely generated. For $n \in \mathbb{Z}_{+}, s \in I$, define $\phi_{n, s}: G \rightarrow \mathbb{R}$ by

$$
\phi_{n, s}(x)= \begin{cases}\xi_{n}(x) & \text { if } x \in A_{\mu} s \\ 0 & \text { otherwise }\end{cases}
$$

Then

$$
\xi_{n}=\sum_{s \in I} \phi_{n, s}
$$

Since $A_{\mu}$ is a subgroup, translation by $k^{-1} \in A_{\mu}$ on the left preserves the right cosets. Hence

$$
k \cdot \xi_{n}=\sum_{s \in I} k \cdot \phi_{n, s}
$$


We will now construct a sequence of unit vectors in $\ell^{2}\left(A_{\mu}\right)$ that are almost invariant. For $n \in \mathbb{Z}_{+}, s \in I$, define $\psi_{n, s}: A_{\mu} \rightarrow \mathbb{R}$ by $\psi_{n, s}(h)=\phi_{n, s}(h s)=\xi_{n}(h s)$, where $h \in A_{\mu}$. Then $\psi_{n, s} \in \ell^{2}\left(A_{\mu}\right)$ since $\sum_{h \in A_{\mu}} \psi_{n, s}(h)=\sum_{h \in A_{\mu}} \xi_{n}(h s)$ is finite. We also have that the norm of $\psi_{n, s}$ in $\ell^{2}\left(A_{\mu}\right)$ is equal to the norm of $\phi_{n, s}$ in $\ell^{2}(G)$. We denote this norm by $a_{n, s}$. Note $\sum_{s \in I}\left(a_{n, s}\right)^{2}=\left\|\xi_{n}\right\|_{2}^{2}=1$.

Putting all this together, we have

$$
\begin{aligned}
\left\|k \cdot \xi_{n}-\xi_{n}\right\|_{2}^{2} & =\left\|\sum_{s \in I} k \cdot \phi_{n, s}-\sum_{s \in I} \phi_{n, s}\right\|_{2}^{2} \\
& =\left\|\sum_{s \in I}\left(k \cdot \phi_{n, s}-\phi_{n, s}\right)\right\|_{2}^{2} \\
& =\sum_{x \in G} \sum_{s \in I}\left[\left(k \cdot \phi_{n, s}-\phi_{n, s}\right)(x)\right]^{2} \\
& =\sum_{s \in I} \sum_{x \in G}\left[\left(k \cdot \phi_{n, s}-\phi_{n, s}\right)(x)\right]^{2} \\
& =\sum_{s \in I} \sum_{y \in A_{\mu}}\left[\left(k \cdot \phi_{n, s}-\phi_{n, s}\right)(y s)\right]^{2}
\end{aligned}
$$

(since $\phi_{n, s}$ is zero outside the $s$-coset)

$$
=\sum_{s \in I}\left(a_{n, s}\right)^{2} \sum_{y \in A_{\mu}}\left[\left(k \cdot \frac{\phi_{n, s}}{a_{n, s}}-\frac{\phi_{n, s}}{a_{n, s}}\right)(y s)\right]^{2} .
$$

Now if, for all $s \in I$, we have

$$
\sum_{y \in A_{\mu}}\left[\left(k \cdot \frac{\phi_{n, s}}{a_{n, s}}-\frac{\phi_{n, s}}{a_{n, s}}\right)(y s)\right]^{2} \geq \epsilon,
$$

then the above equation becomes

$$
\left\|k \cdot \xi_{n}-\xi_{n}\right\|_{2}^{2} \geq \epsilon \sum_{s \in I}\left(a_{n, s}\right)^{2}=\epsilon .
$$

Therefore, $\left\|k \cdot \xi_{n}-\xi_{n}\right\|_{2}^{2}<\epsilon$ implies there exists $s$ such that

$$
\sum_{y \in A_{\mu}}\left[\left(k \cdot \frac{\phi_{n, s}}{a_{n, s}}-\frac{\phi_{n, s}}{a_{n, s}}\right)(y s)\right]^{2}<\epsilon .
$$

Since $\left\|k \cdot \xi_{n}-\xi_{n}\right\|_{2}^{2}$ limits to zero for every $k \in A_{\mu}$, there exists a sequence $s_{n}$ for which

$$
\sum_{y \in A_{\mu}}\left[\left(k \cdot \frac{\phi_{n, s_{n}}}{a_{n, s_{n}}}-\frac{\phi_{n, s_{n}}}{a_{n, s_{n}}}\right)(y s)\right]^{2} \rightarrow 0 \quad \text { for every } k \in A_{\mu} .
$$


Rewriting in terms of corresponding functions in $\ell^{2}\left(A_{\mu}\right)$,

$$
\sum_{y \in A_{\mu}}\left[\left(k \cdot \frac{\psi_{n, s_{n}}}{a_{n, s_{n}}}-\frac{\psi_{n, s_{n}}}{a_{n, s_{n}}}\right)(y)\right]^{2}=\left\|k \cdot \frac{\psi_{n, s_{n}}}{a_{n, s_{n}}}-\frac{\psi_{n, s_{n}}}{a_{n, s_{n}}}\right\|_{2}^{2} \rightarrow 0,
$$

so $\frac{\psi_{n, s_{n}}}{a_{n, s_{n}}}$ supplies a sequence of almost invariant unit vectors in $\ell^{2}\left(A_{\mu}\right)$, and $A_{\mu}$ is amenable.

That $A_{\mu}$ is an amenable subgroup does not preclude it being trivial for all nonamenable $G$, nor does it guarantee that $A_{\mu}$ reflects any of the underlying structure of $G$. The next result shows that in many cases, $A_{\mu}$ is an interesting non-trivial subgroup.

Recall that the elliptic radical of a finitely generated group $G$ is the largest normal, locally finite subgroup of $G$ (see, for example, [4]). It is the group generated by all finite normal subgroups of $G$ and is contained in the amenable radical, the largest amenable normal subgroup. We now prove a result which implies that the elliptic radical is contained in $A_{\mu}$.

Theorem 4.3. $A_{\mu}$ contains every finite normal subgroup of $G$. In particular, the elliptic radical is contained in $A_{\mu}$.

Proof. Let $F$ be a finite normal subgroup of $G$. Since the support of $\mu$ generates $G, F$ is finite and $\mu$ is aperiodic, we have $\mu^{*|F|}(f)$ is non-zero for all $f \in F$. Setting $\kappa=\mu^{*|F|}$, we have $F \subseteq \operatorname{supp}(\kappa)$, and $A_{\mu}=A_{\kappa}$ by Lemma 3.4.

Let $S=\operatorname{supp}(\kappa)$. Then each walk $\left(g_{0}=e, g_{1}, \ldots\right)$ induced by $\kappa$ corresponds uniquely to a sequence $\left(\left(h_{0}, f_{0}\right),\left(h_{1}, f_{1}\right), \ldots\right)$, where $h_{0}=f_{0}=e, h_{i} \in\langle S \backslash F\rangle$, $f_{i} \in F$ and $g_{i}=h_{i} f_{i}$ defined by the following process: if $g_{n}=g_{n-1} x, x \in \operatorname{supp}(\kappa)$, then

$$
\left(h_{n}, f_{n}\right)= \begin{cases}\left(h_{n-1}, f_{n-1} x\right), & x \in F, \\ \left(h_{n-1} x, x^{-1} f_{n-1} x\right), & x \in S \backslash F .\end{cases}
$$

Define the measure $\phi:\langle S \backslash F\rangle \rightarrow \mathbb{R}$ by

$$
\phi(x)= \begin{cases}\kappa(F), & x=e, \\ \kappa(x), & x \in S \backslash F .\end{cases}
$$

Then $\phi^{* n}$ is the distribution of the first coordinate after $n$ steps.

The process on the second coordinate is a Markov chain on the state space $F$, where each move corresponds either to a right multiplication by $x \in F$ or to a conjugation by some element of $x \in S \backslash F$, each with probability $\kappa(x)$. Let $\tau_{n}$ denote 
the distribution of the second coordinate after $n$ steps. We will prove that $\tau_{n}$ approaches the uniform distribution of $F$ using standard Markov chain theory (see for example [6] for further information).

Let $\operatorname{Pr}(f \rightarrow g)$ be the probability of moving from state $f$ to $g$ in one step of the Markov chain. If the step from $f$ to $g$ is a conjugation by $x$ (i.e., $g=x^{-1} f x$ ), then $f=x g x^{-1}$, so $\operatorname{Pr}(f \rightarrow g)=\operatorname{Pr}(g \rightarrow f)$ since $\kappa$ is symmetric. Otherwise, the step is induced by right multiplication, and clearly $\operatorname{Pr}(f \rightarrow g)=\operatorname{Pr}(g \rightarrow f)$ (since $g=f x$ only if $f=g x^{-1}$ ). It follows that the Markov chain satisfies the detailed balance condition for the uniform measure $\pi=\frac{1}{|F|}$, i.e.,

$$
\pi(f) \operatorname{Pr}(f \rightarrow g)=\pi(g) \operatorname{Pr}(g \rightarrow f),
$$

and so $\pi$ is a stationary distribution on $F$, that is,

$$
\pi(f)=\sum_{y \in F} \pi(y) \operatorname{Pr}(y \rightarrow f) .
$$

Since $e \in \operatorname{supp}(\kappa)$, the Markov process on $F$ is aperiodic, and since $F$ is finite, the process is irreducible. Then, by the fundamental theorem of Markov chains (see, for example, [6, Theorem 3.12]), $\tau_{n}$ converges to the unique stationary distribution $\pi$.

Now consider an $n$-step walk of the walk motivated by $\kappa$, which ends at some $f \in F$. We have

$$
\kappa^{* n}(f)=\sum_{g \in F} \phi^{* n}(g) \cdot \tau_{n}\left(g^{-1} f\right)
$$

since to end at $f$, we must have first coordinate $g$ and second coordinate $g^{-1} f \in F$. Then

$$
\begin{aligned}
\lim _{n \rightarrow \infty} \frac{\kappa^{* n}(f)}{\kappa^{* n}(e)} & =\lim _{n \rightarrow \infty} \frac{\sum_{g \in F} \phi^{* n}(g) \tau_{n}\left(g^{-1} f\right)}{\sum_{g \in F} \phi^{* n}(g) \tau_{n}\left(g^{-1}\right)} \\
& =\lim _{n \rightarrow \infty} \frac{\sum_{g \in F} \phi^{* n}(g) \pi\left(g^{-1} f\right)}{\sum_{g \in F} \phi^{* n}(g) \pi\left(g^{-1}\right)}=1
\end{aligned}
$$

since $\pi$ is the uniform distribution on $F$, and so $F \subseteq A_{\kappa}=A_{\mu}$.

Theorem 4.3 is notable for two reasons. Firstly, it shows that, whenever a finitely generated group contains a finite normal subgroup, $A_{\mu}$ is non-trivial. Secondly, this result is independent of $\mu$. 


\section{Examples}

Recall that non-abelian free groups have no non-trivial amenable normal subgroups. That is, the amenable radical is trivial.

Lemma 5.1. Let $F_{d}$ be the free group of rank $d \geq 2$ with free basis generators including $a, b$, and let $\mu$ be a symmetric, aperiodic measure whose support generates $F_{d}$ satisfying $\mu(e)>0$ and $\mu(a)=\mu(b)>0$. Then $A_{F_{d}, \mu}$ is trivial.

Proof. Let $u \in\left\{a^{ \pm 1}, b^{ \pm 1}\right\}^{+}$. If $u \in A_{\mu}$, then by interchanging $a^{ \pm 1}$ with $b^{ \pm 1}$, we obtain a word $v$ that also lies in $A_{\mu}$ by symmetry of the measure with respect to the generators $a, b$. If $u, v$ are not powers of the same element, in which case they generate a free group of rank 2 , and since $A_{\mu}$ is an amenable subgroup, it must be trivial. Otherwise, if $u, v$ generate a cyclic group, choose instead to replace $a^{ \pm 1}$ by $b^{\mp 1}$.

Lemma 5.2. Suppose $G, H$ are finitely generated groups with symmetric, aperiodic probability measures $\phi$ and $\psi$ respectively whose supports generate $G$ and $H$ respectively. Recall that the product measure $\mu$ on $G \times H$ is defined by

$$
\mu(x, y)=\phi(x) \psi(y) .
$$

Then

$$
A_{G \times H, \mu}=A_{G, \phi} \times A_{H, \psi} .
$$

Proof. To prove this, we first note that $\mu^{* n}(x, y)=\phi^{* n}(x) \psi^{* n}(y)$. This may be shown inductively. It is true for $n=1$ by definition, and

$$
\mu^{* n}(x, y)=\phi^{* n}(x) \psi^{* n}(y)
$$

implies

$$
\begin{aligned}
\mu_{n+1}(x, y) & =\sum_{(g, h) \in G \times H} \mu^{* n}(g, h) \mu\left(g^{-1} x, h^{-1} y\right) \\
& =\sum_{g \in G} \sum_{h \in H}\left[\phi^{* n}(g) \psi^{* n}(h)\right]\left[\phi\left(g^{-1} x\right) \psi\left(h^{-1} y\right)\right] \\
& =\sum_{g \in G} \sum_{h \in H}\left[\phi^{* n}(g) \phi\left(g^{-1} x\right)\right]\left[\psi^{* n}(h) \psi\left(h^{-1} y\right)\right] \\
& =\sum_{g \in G} \phi^{* n}(g) \phi\left(g^{-1} x\right) \sum_{h \in H} \psi^{* n}(h) \psi\left(h^{-1} y\right) \\
& =\phi_{n+1}(x) \psi_{n+1}(y) .
\end{aligned}
$$


Thus

$$
\begin{aligned}
\lim _{n \rightarrow \infty} \frac{\mu^{* n}(g, h)}{\mu^{* n}\left(e_{G}, e_{H}\right)} & =\lim _{n \rightarrow \infty} \frac{\phi^{* n}(g) \psi^{* n}(h)}{\phi^{* n}\left(e_{G}\right) \psi^{* n}\left(e_{H}\right)} \\
& =\lim _{n \rightarrow \infty} \frac{\phi^{* n}(g)}{\phi^{* n}\left(e_{G}\right)} \lim _{n \rightarrow \infty} \frac{\psi^{* n}(h)}{\psi^{* n}\left(e_{H}\right)}
\end{aligned}
$$

from which the result follows.

Example 5.3. Let $F_{d}$ be the free group of rank $d \geq 2$ with free basis generators including $a, b$, and let $\phi$ be a symmetric, aperiodic measure whose support generates $F_{d}$ satisfying $\phi(e)>0$ and $\phi(a)=\phi(b)>0$. Let $H$ be an amenable group with good measure $\psi$, and let $\mu$ be the product measure on $F_{d} \times H$. Then $A_{F_{d} \times H, \mu}=H$, which is exactly the amenable radical of $F_{d} \times H$.

In light of these examples and the fact that $A_{\mu}$ contains the elliptic radical, one might ask whether $A_{\mu}$ is in fact always the amenable radical. If so, this would imply for one thing that the set $A_{\mu}$ is invariant under choice of measure. It turns out that this is not the case - in the next section, we give an example where the amenable radical is trivial but $A_{\mu}$ is not. Moreover, we show that $A_{\mu}$ depends on the choice of measure.

\section{Dependence on the measure}

Proposition 6.1. Let $G$ be a finitely generated group with a finite subgroup $F$. Then there exists a symmetric, aperiodic probability measure $\phi$ on $G$ whose support generates $G$ such that $F \subset A_{\phi}$.

Proof. Take $\psi=\pi_{F} * \mu * \pi_{F}$, where $\pi_{F}$ is the uniform measure on $F$. Then

$$
\phi(x)=\frac{1}{|F|^{2}} \sum_{f_{1}, f_{2} \in F} \mu\left(f_{1} x f_{2}\right),
$$

which is symmetric, $\phi(e) \geq \frac{1}{|F|^{2}} \mu(e)>0$ and $\operatorname{supp}(\phi) \supseteq \operatorname{supp}(\mu)$. For $f \in F$, $x \in G$, we also have $\phi(f x)=\phi(x)$, so

$$
\begin{aligned}
\psi^{* n}(f) & =\sum_{g \in G} \psi(g) \psi^{* n-1}\left(g^{-1} f\right) \\
& =\sum_{x \in G} \psi\left(f^{-1} g\right) \psi^{* n-1}\left(g^{-1} f\right) \\
& =\psi^{* n}(e),
\end{aligned}
$$

so $F \subset A_{\psi}$. 
Corollary 6.2. There exists a finitely generated group $G$ and symmetric, aperiodic probability measures $\mu, \tau$ on $G$ whose support generates $G$ so that $A_{G, \mu} \neq A_{G, \tau}$.

Proof. Consider the free product $G=\left\langle a \mid a^{2}=1\right\rangle *\left\langle b \mid b^{3}=1\right\rangle$. By Proposition 6.1, there are measures $\mu, \tau$ so that $a \in A_{\mu}$ and $b \in A_{\tau}$. If $A_{\mu}=A_{\tau}$, then $A_{\mu}=\langle a, b\rangle=G$, which is a contradiction since $G$ is not amenable. Other examples are readily constructed from free products of finite groups.

The same example also gives the following:

Corollary 6.3. There exists a finitely generated group $G$ and a symmetric, aperiodic probability measure $\mu$ on $G$ whose support generates $G$ so that $A_{\mu}$ is not equal to the amenable radical.

Proof. Since $C_{2} * C_{3}$ contains finite subgroups, we may use the arguments from Proposition 6.1 to construct a measure $\mu$ for which $A_{\mu}$ is non-trivial. However, $C_{2} * C_{3}$ has a trivial amenable radical. This follows from the fact that it is $C^{*}$ simple [1], or by considering the action of the group on a tree. If $N$ is a normal amenable subgroup of a group acting on a tree, then by normality and the Tits alternative, it fixes all vertices in $G / A$ (or $G / B$ ), or all edges, or a $G$-orbit of ends. Since the $G$-action on the space of ends is minimal, this implies in all three cases that $N$ is trivial.

In particular, $A_{\mu}$ is not always normal.

\section{Connection to the amenable radical}

In all cases considered, $A_{\mu}$ always contains the amenable radical. If this were true for all measures $\mu$, the next results would give a way to directly link the amenable radical with random walk distributions.

Lemma 7.1. Let $G$ be a finitely generated group, and let $\mu$ be a symmetric, aperiodic probability measure on $G$ whose support generates $G$. Define a measure $\mu_{g}: G \rightarrow \mathbb{R}$ by $\mu_{g}(x)=\mu\left(g^{-1} x g\right)$ for each $x \in G$. Then $\mu_{g}$ is a symmetric, aperiodic probability measure on $G$ whose support generates $G$, and $A_{\mu_{g}}=g A_{\mu} g^{-1}$.

Proof. We have

$$
\begin{array}{r}
\mu_{g}\left(x^{-1}\right)=\mu\left(g^{-1} x^{-1} g\right)=\mu\left(g^{-1} x g\right)=\mu_{g}(x), \\
\mu_{g}(e)=\mu(e)>0 \text { and } \operatorname{supp}\left(\mu_{g}\right)=g^{-1} \operatorname{supp}(\mu) g=G .
\end{array}
$$


For $y \in G$,

$$
\begin{aligned}
\mu_{g}^{* 2}(y) & =\sum_{x \in G} \mu_{g}(x) \mu_{g}\left(x^{-1} y\right) \\
& =\sum_{x \in G} \mu\left(g^{-1} x g\right) \mu\left(g^{-1} x^{-1} y g\right) \\
& =\sum_{h \in G} \mu\left(g^{-1} h\right) \mu\left(h^{-1} y g\right) \\
& =\mu^{* 2}\left(g^{-1} y g\right) .
\end{aligned}
$$

Using an inductive argument, it is clear that

$$
\mu_{g}^{* n}(y)=\mu^{* n}\left(g^{-1} y g\right) \text {. }
$$

Now

$$
\begin{aligned}
x \in A_{\mu^{g}} & \Longleftrightarrow \lim _{n \rightarrow \infty} \frac{\mu_{g}^{* n}(x)}{\mu_{g}^{* n}(e)}=1 \\
& \Longleftrightarrow \lim _{n \rightarrow \infty} \frac{\mu^{* n}\left(g^{-1} x g\right)}{\mu^{* n}(e)}=1 \\
& \Longleftrightarrow g^{-1} x g \in A_{\mu} \\
& \Longleftrightarrow x \in g A_{\mu} g^{-1} .
\end{aligned}
$$

Proposition 7.2. Let $G$ be a finitely generated group. If $x \in G$ does not belong to the amenable radical, then for any symmetric, aperiodic probability measure $\mu$ on $G$ whose support generates $G$, there exists $g \in G$ such that $x \notin A_{\mu_{g}}$.

Proof. Suppose for contradiction that $x \in G$ belongs to $A_{\mu}$ for every symmetric, aperiodic probability measure $\mu$ on $G$ whose support generates $G$. Then for some fixed $\mu$, by the previous lemma, we have $x \in A_{\mu_{g}}=g A_{\mu} g^{-1}$ for all $g \in G$. Thus

$$
x \in \bigcap_{g \in G} g A_{\mu} g^{-1},
$$

which is a normal amenable subgroup; hence $x$ belongs to the amenable radical.

Corollary 7.3. Let $\mathcal{A}_{G}$ denote the amenable radical of $G$, and let $\mathcal{M}_{G}$ be the set of all symmetric, aperiodic probability measures on $G$ whose support generates $G$. If $\mathcal{A}_{G} \subseteq A_{\mu}$ for every $\mu \in \mathcal{M}_{G}$, then $\mathcal{A}_{G}=\bigcap_{\mu} A_{\mu \in \mathcal{M}_{G}}$.

We close by formulating two conjectures. 
Conjecture 7.4. Let $G$ be a finitely generated group. Then for any symmetric, aperiodic probability measure $\mu$ on $G$ whose support generates $G$, the subgroup $A_{\mu}$ contains the amenable radical.

Even more desirable would be the following:

Conjecture 7.5. Let $G$ be a finitely generated group. Then there exists some $\mu$ such that $A_{\mu}$ is the amenable radical.

Acknowledgments. The authors wish to thank Alain Valette, Sasha Fish, Vadim Kaimanovich, Narutaka Ozawa, Anne Thomas, George Willis and the anonymous reviewer for helpful suggestions, references and feedback.

\section{Bibliography}

[1] C. A. Akemann, Operator algebras associated with Fuchsian groups, Houston J. Math. 7 (1981), no. 3, 295-301.

[2] A. Avez, Limite de quotients pour des marches aléatoires sur des groupes, C. R. Acad. Sci. Paris Sér. A-B 276 (1973), A317-A320.

[3] B. Bekka, P. de la Harpe and A. Valette, Kazhdan's Property (T), New Math. Monogr. 11, Cambridge University, Cambridge, 2008.

[4] P.-E. Caprace and N. Monod, Fixed points and amenability in non-positive curvature, Math. Ann. 356 (2013), no. 4, 1303-1337.

[5] M. M. Day, Means on semigroups and groups, Bull. Amer. Math. Soc. 55 (1949), 1054-1055.

[6] M. Elder, A. Rechnitzer and E. J. Janse van Rensburg, Random sampling of trivial words in finitely presented groups, Exp. Math. 24 (2015), no. 4, 391-409.

[7] A. Erschler, Almost invariance of distributions for random walks on groups, preprint (2016), https://arxiv.org/abs/1603.01458.

[8] A. Gournay, Amenable groups, Notes for short course at CIRM Luminy (2014).

[9] V. A. Kămanovich and A. M. Vershik, Random walks on discrete groups: Boundary and entropy, Ann. Probab. 11 (1983), no. 3, 457-490.

[10] C. Pittet and L. Saloff-Coste, On the stability of the behavior of random walks on groups, J. Geom. Anal. 10 (2000), no. 4, 713-737.

[11] H. Reiter and J. D. Stegeman, Classical Harmonic Analysis and Locally Compact Groups, 2nd ed., London Math. Soc. Monogr. Ser. (N. S.) 22, Clarendon Press, New York, 2000. 
[12] A. Thomas, The Banach-Tarski paradox and amenability, Course Notes, University of Sydney, 2011.

[13] J. G. F. Wan, Random walks, elliptic integrals and related constants, Bull. Aust. Math. Soc. 89 (2014), no. 1, 166-167.

[14] W. Woess, Random walks on infinite graphs and groups - a survey on selected topics, Bull. Lond. Math. Soc. 26 (1994), no. 1, 1-60.

Received December 20, 2017; revised November 11, 2018.

\section{Author information}

Murray Elder, School of Mathematical and Physical Sciences,

University of Technology Sydney, Ultimo NSW 2007, Australia.

E-mail: murrayelder@gmail. com

Cameron Rogers, Launceston Church Grammar School, Mowbray Heights, Launceston TAS 7248, Australia.

E-mail: cameron.m.rogers@gmail.com 